\title{
Post-Splenectomy Portal Venous Thrombosis in Cirrhotic Patients: An Observational Clinical Trial
}

\author{
Hamdy Sedky Abd Allah*, Mohamad Hamdy Abo-Ryia, Sherif Abd-Al Fattah Saber \\ General Surgery Department, Faculty of Medicine, Tanta University, Tanta, Egypt \\ Email address: \\ Hamdy.abdallah@med.tanta.edu.eg (H. S. A. Allah) \\ ${ }^{*}$ Corresponding author \\ To cite this article: \\ Hamdy Sedky Abd Allah, Mohamad Hamdy Abo-Ryia, Sherif Abd-Al Fattah Saber. Post-Splenectomy Portal Venous Thrombosis in \\ Cirrhotic Patients: an Observational Clinical Trial. Journal of Surgery. Vol. 5, No. 6, 2017, pp. 105-110. doi: 10.11648/j.js.20170506.14
}

Received: September 22, 2017; Accepted: October 4, 2017; Published: November 12, 2017

\begin{abstract}
Background: Post-splenectomy portal venous thrombosis (PS-PVT) carries multiple threats to patients' lives. Different variables were identified as risk factors for PS-PVT in cirrhotic patients. The aim of this study was to prospectively assess the incidence, risk factors, clinical presentation and treatment outcomes of PS-PVT in cirrhotic patients. Patients and methods: Sixty cirrhotic patients of Child class A submitted to open splenectomy were observed, both clinically and by Duplex ultrasound (US) examination, for the development of PS-PVT. Results: Overall, 17 patients (28.3\%) developed PS-PVT at a median interval of 4.5 days (21 hours-7 days) post-splenectomy. Univariate analysis showed that lower preoperative platelet count $(\mathrm{P}<0.0460)$ and white blood cell $(\mathrm{WBC})$ count $(\mathrm{P}<0.0001)$ and wider splenic vein diameter $(\mathrm{SVD})(\mathrm{P}<0.0001)$ correlated with PS-PVT. Multivariate analysis identified lower preoperative WBC count [odds ratio (OR): $0.651,95 \%$ confidence interval (CI): 0.245-0.893, P<0.005] and wider SVD (OR: 2.383, 95\% CI: 1.558-3.646., P $<0.001$ ) as independent risk factors of PSPVT. While 16 out of the 17 patients (94\%) who had these 2 risk factors developed PS-PVT, only 1 out of the 43 patients (2.3\%) who didn't have the same risk factors developed thrombosis. All 17 patients had complete resolution of their thrombosis on anticoagulation therapy within 3-6 months without complications or mortality. Conclusion: PVT is a common complication of splenectomy in cirrhotic patients. Patients with low WBC count and wide SVD are highly susceptible to develop this complication mandating close observation from the $1^{\text {st }} \mathrm{PO}$ day and immediate anticoagulation after diagnosis.
\end{abstract}

Keywords: Splenectomy, Portal Vein Thrombosis, Cirrhosis, Risk Factors

\section{Introduction}

Egypt has the highest prevalence of hepatitis $\mathrm{C}$ virus (HCV) infection in the world $[1,2]$ reaching $22 \%$ in those aged 55-59 years in 2015 [3]. Liver cirrhosis develops in $48 \%$ of patients having co-infection with schistosomiasis [4]. In a community-based study in Nile Delta, 3\% of tested subjects were cirrhotic and $67 \%$ of cirrhotic patients had portal hypertension [5].

Cirrhotic patients with portal hypertension frequently require splenectomy for various indications, including hypersplenism, thrombocytopenia developing before or during $\mathrm{HCV}$ treatment, thrombocytopenia in patients with hepatocellular carcinoma (HCC) and as a part of Hassab operation [6-9].

With an incidence of $16-52.6 \%$ in cirrhotic patients [10-
14], post-splenectomy portal venous thrombosis (PS-PVT) carries multiple threats to patients' lives including upper gastrointestinal bleeding, ischemic bowel necrosis, liver cell failure and refractory ascites $[15,16]$.

Since symptoms, such as abdominal pain and fever are non-specific and PS-PVT can easily develop without any symptoms, identification of high-risk patients is essential to allow starting anticoagulation immediately once the diagnosis is made $[17,18]$. In one study, all patients treated within 10 days of splenectomy had complete resolution of the clot, in comparison to no patient whose treatment was initiated more than 10 days after splenectomy [19].

Different variables were identified as risk factors for PSPVT in cirrhotic patients including a wider preoperative portal vein $(\mathrm{PV})$ diameter $[10,13]$ or splenic vein diameter (SVD) $[12,14,20,21]$, preoperative low platelet [14] or white blood cell (WBC) counts [12], prolonged prothrombin 
time [10], decreased antithrombin-III activity [20], postoperative thrombocytosis [10] and age $>50$ years [13]. The aim of this study was to prospectively assess the complication of PS-PVT in cirrhotic patients regarding its incidence, risk factors, clinical presentation and treatment.

\section{Patients and Methods}

\subsection{Study Design}

This observational clinical trial was conducted at the Gastrointestinal and Laparoscopic Surgery Unit, General Surgery Department, Tanta University, Tanta, Egypt during the period from March 2008 to March 2016. Cirrhotic patients of Child class A submitted to open splenectomy for symptomatic splenomegaly, 2ry hypersplenism or thrombocytopenia (Platelet count $<50 \times 10^{3} / \mathrm{CC}$ ) were included in the study while cirrhotic patients of Child class B or $\mathrm{C}$, patients with preoperative PVT and patients scheduled for splenectomy for other indications were excluded. An informed written consent was obtained from every patient and the study protocol was approved by the "Research Ethics Committee" of the Faculty of Medicine, Tanta University.

Preoperatively, the age, sex, hepatitis virus status, hemoglobin $(\mathrm{Hb})$ concentration, platelet count, WBC count and international normalized ratio (INR) were collected. Preoperative Duplex Doppler ultrasound (US) for exclusion of PVT and measuring the SV diameter was performed. If not performed before, upper endoscopy was performed for control of esophago-gastric varices if indicated. No prophylactic anticoagulants were used. Splenectomy was performed through a left subcostal incision. Operative time and intraoperative blood product transfusion were recorded. Duplex Doppler US was performed at postoperative (PO) days 1,3,7 and 30. When PS-PVT was detected, its time, distribution and extent were recorded and therapeutic dose of heparin was started followed by oral anticoagulant. WBC and Platelet counts were measured at PO days 3, 7 and 30. PS-PVT was defined as either complete or partial thrombosis of the PV trunk, the PV branches or the superior mesenteric vein (SMV). Clinical signs and symptoms suggestive of PSPVT were looked for and recorded. After discharge, Duplex Doppler US was repeated every month for a maximum of 6 months or till complete recanalization of the thrombosed vein.

\subsection{Statistical Analysis}

The primary end-point was the development of PS-PVT and secondary end-points included complications of PVT, complications of treatment and mortality. Data were tabulated and analyzed using SPSS statistics software package version 20. Continuous data were expressed as range, median and mean and were compared using the twotailed, non-paired Student's $t$-test. Categorical data were expressed as frequencies and were compared using Fisher's exact test. Only variables that correlated positively with PSPVT on univariate analysis were considered for multivariate analysis using the logistic regression model. $\mathrm{P}$ value $<0.05$ was considered statistically significant.

\section{Results}

The study population included 60 patients who were divided into 2 groups; group (A) included patients who developed PS-PVT and group (B) included patients who did not.

\subsection{Pre-Operative Data [Table 1]}

In group (A), there were 10 males (59\%) and 7 females (41\%) with a median age of 44 years (range 21-67, mean 40.9 years). The indications of surgery included symptomatic splenomegaly, 2ry hypersplenism and thrombocytopenia before or during HCV treatment in 4 (23.5\%), 9 (53\%) and 4 $(23.5 \%)$ patients respectively. All patients had thrombocytopenia and the median preoperative platelet count was $45 \times 10^{3} / \mathrm{CC}$ (range $22-145$, mean $60.2 \times 10^{3} / \mathrm{CC}$ ), the median preoperative WBC count was $1.81 \times 10^{3} / \mathrm{CC}$ (range 1.4-3.3, mean $1.9 \times 10^{3} / \mathrm{CC}$ ) and the median preoperative SVD was $14.3 \mathrm{~mm}$ (range 6-16, mean $13.5 \mathrm{~mm}$ ).

In group (B), there was 25 males (58.1\%) and 18 females $(41.9 \%)$ with a median age of 41.2 years (range $23-60$, mean 40.6 years). The indications of surgery included symptomatic splenomegaly, 2ry hypersplenism and thrombocytopenia in the course of HCV treatment in $8(18.6 \%), 25(58.1 \%)$ and $10(23.3 \%)$ patients respectively. Thirty five patients $(81.4 \%)$ had thrombocytopenia and the median preoperative platelet count was $82.5 \times 10^{3} / \mathrm{CC}$ (range 25-240, mean $\left.88.48 \times 10^{3} / / \mathrm{CC}\right)$, the median preoperative WBC count was $6.4 \times 10^{3} / / \mathrm{CC}$ (range $3.5-9.3$, mean $6 \times 10^{3} / \mathrm{CC}$ ) while the median preoperative SVD was $8.5 \mathrm{~mm}$ (range 5-14, mean 6.5 $\mathrm{mm})$.

Table 1. Pre-Operative Patients' Data.

\begin{tabular}{|c|c|c|c|c|c|}
\hline \multirow{2}{*}{ Variable } & \multicolumn{2}{|c|}{ Group A $(\mathrm{No}=17)$} & \multicolumn{2}{|c|}{ Group $B(\mathrm{No}=43)$} & \multirow{2}{*}{$\boldsymbol{P}$} \\
\hline & No & $\%$ & No & $\%$ & \\
\hline \multicolumn{6}{|l|}{ Sex } \\
\hline Male & 10 & 59 & 25 & 58.1 & 1.0000 \\
\hline Female & 7 & 41 & 18 & 41.9 & 1.0000 \\
\hline Median age (years) & \multicolumn{2}{|c|}{40.8} & \multicolumn{2}{|c|}{41.2} & 0.986 \\
\hline \multicolumn{6}{|l|}{ HCV status } \\
\hline Active infection & 9 & 53 & 28 & 65 & 0.8142 \\
\hline Treated & 8 & 47 & 14 & 32.5 & 0.5896 \\
\hline Median INR & \multicolumn{2}{|c|}{1.4} & \multicolumn{2}{|c|}{1.3} & 0.3467 \\
\hline Median $\mathrm{Hb}$ concentration $(\mathrm{gm} / \mathrm{dl})$ & \multicolumn{2}{|c|}{9.1} & \multicolumn{2}{|c|}{9.5} & 0.8425 \\
\hline
\end{tabular}




\begin{tabular}{|c|c|c|c|c|c|}
\hline \multirow{2}{*}{ Variable } & \multicolumn{2}{|c|}{ Group A $\left(\mathrm{No}_{0}=17\right)$} & \multicolumn{2}{|c|}{ Group B $(\mathrm{No}=43)$} & \multirow{2}{*}{$\boldsymbol{P}$} \\
\hline & No & $\%$ & No & $\%$ & \\
\hline Median platelet count $\left(\mathrm{x} 10^{3} / \mathrm{CC}\right)$ & \multicolumn{2}{|c|}{45} & \multicolumn{2}{|c|}{82.5} & 0.0460 \\
\hline Median WBC count $\left(\times 10^{3} / \mathrm{CC}\right)$ & \multicolumn{2}{|c|}{1.81} & \multicolumn{2}{|c|}{6.4} & 0.0001 \\
\hline Median SVD $(\mathrm{mm})$ & \multicolumn{2}{|c|}{14.3} & \multicolumn{2}{|c|}{8.5} & 0.0001 \\
\hline \multicolumn{6}{|l|}{ Indications of surgery } \\
\hline Symptomatic splenomegaly & 4 & 23.5 & 8 & 18.6 & 0.7366 \\
\hline 2ry hypersplenism & 9 & 53 & 25 & 58.1 & 1.0000 \\
\hline Thrombocytopenia & 4 & 23.5 & 10 & 23.3 & 1.0000 \\
\hline
\end{tabular}

HCV: Hepatitis C virus, Hb: Hemoglobin, WBC: white blood cell, INR: international normalized ratio, SVD: splenic vein diameter.

\subsection{Operative Data [Table 2]}

In group (A), the median number of units of packed red blood cells (RBC), blood platelets and fresh frozen plasma (FFP) per patient was 1,3 and 1.5 units respectively. The median operative time was 84 minutes (range 95-120, mean
89 minutes). In group (B), the median number of units of packed red blood cells, blood platelets and FFP per patient was $1.3,3$ and 2 units respectively. The median operative time was 77 minutes (range 80-130, mean 78 minutes).

Table 2. Operative Data.

\begin{tabular}{|c|c|c|c|}
\hline Variable & Group A $(\mathrm{No}=17)$ & Group B $(\mathrm{No}=43)$ & $\boldsymbol{P}$ \\
\hline \multicolumn{4}{|c|}{ Blood products transfusion (median No. of units/patient) } \\
\hline Packed RBC & 1 & 1.3 & 0.4997 \\
\hline Blood platelets & 3 & 3 & 0.3570 \\
\hline Median operative time (minutes) & 84 & 77 & 0.3347 \\
\hline
\end{tabular}

RBC: red blood cells, FFP: fresh frozen plasma.

\subsection{Post-Operative Data [Table 3]}

Overall, 17 patients $(17 / 60 ; 28.3 \%)$ developed PS-PVT at a median interval of 4.5 days post-splenectomy. PS-PVT was diagnosed 21 hours after surgery in 1 patient $(1 / 17 ; 5.9 \%)$, at the $3^{\text {rd }} \mathrm{PO}$ day in 9 patients $(9 / 17 ; 52.9 \%)$ and at the $7^{\text {th }} \mathrm{PO}$ day in 7 patients $(7 / 17 ; 41.2 \%)$.

The preoperative, operative and PO data of patients in both groups, including age, sex, $\mathrm{HCV}$ status, preoperative $\mathrm{Hb}$ concentration, WBC count, platelet count, INR, SVD, indications of surgery, operative time, intra-operative $\mathrm{RBC}$, FFP and platelet transfusion, PO WBC and platelet count, were compared. On Univariate analysis, 3 variables correlated with PS-PVT including lower preoperative platelet count $(\mathrm{p}<0.0460)$, lower preoperative WBC count $(\mathrm{P}<0.0001)$ and wider SVD $(\mathrm{P}<0.0001)$. Multivariate analysis identified lower preoperative WBC count [odds ratio (OR): $0.651,95 \%$ confidence interval $(\mathrm{CI}): 0.245-0.893, \mathrm{P}<0.005]$ and wider preoperative SVD (OR: $2.383,95 \%$ CI: $1.558-$ 3.646., $\mathrm{P}<0.001)$ as independent risk factors of PS-PVT. In this study, 17 patients had low WBC count and wide SVD and 16 of them $(16 / 17 ; 94 \%)$ developed PS-PVT. On the other hand, only 1 out of the 43 patients $(1 / 43 ; 2.3 \%)$ who didn't have these 2 variables developed PS-PVT $(\mathrm{P}<0.0001)$. Figure 1

The SV was totally thrombosed in all patients, the PV was totally thrombosed in 13 patients $(13 / 17 ; 76.5 \%)$ and partially thrombosed in 4 patients $(4 / 17 ; 23.5 \%)$ while the SMV was partially thrombosed in 5 patients $(5 / 17 ; 29.4 \%)$ and without thrombosis in the remaining 12 patients $(13 / 17$; $70.6 \%)$.

Table 3. Post-Operative Data.

\begin{tabular}{|c|c|c|c|c|c|}
\hline \multirow{2}{*}{ Variable } & \multicolumn{2}{|c|}{ Group A $(\mathrm{No}=17)$} & \multicolumn{2}{|c|}{ Group B $(\mathrm{No}=43)$} & \multirow{2}{*}{$\boldsymbol{P}$} \\
\hline & No & $\%$ & No & $\%$ & \\
\hline Median PO WBC count $\left(\times 10^{3} / \mathrm{CC}\right)$ & \multicolumn{2}{|c|}{14.29} & \multicolumn{2}{|c|}{12.65} & 0.0012 \\
\hline Median PO platelet count $\left(\times 10^{3} / \mathrm{CC}\right)$ & \multicolumn{2}{|c|}{620} & \multicolumn{2}{|c|}{690} & 0.5000 \\
\hline \multicolumn{6}{|l|}{ Timing of PS-PVT } \\
\hline PO day 1 & 1 & 5.9 & 0 & 0 & \\
\hline PO day 3 & 9 & 52.9 & 1 & 5.9 & \\
\hline PO day 7 & 6 & 35.3 & 0 & 0 & \\
\hline Overall & \multicolumn{4}{|c|}{$12(70.6 \%)$} & \\
\hline Abdominal pain/discomfort & \multicolumn{4}{|c|}{$9(53 \%)$} & \\
\hline Anorexia & \multicolumn{4}{|c|}{$6(35.3 \%)$} & \\
\hline Abdominal distension & \multicolumn{4}{|c|}{$5(29.4 \%)$} & \\
\hline
\end{tabular}

PS-PVT: post-splenectomy portal venous thrombosis, WBC: white blood cell, PO: postoperative.

While 5 out of the 17 patients $(5 / 17 ; 29.4 \%)$ who developed PS-PVT were entirely asymptomatic, 12 patients
$(12 / 17 ; 70.6 \%)$ developed symptoms correlated with the onset of PS-PVT including vague central abdominal 
pain/discomfort in 9 patients $(9 / 17 ; 53 \%)$, anorexia in 6 patients $(6 / 17 ; 35.3 \%)$ and abdominal distension in 5 patients $(5 / 17 ; 29.4 \%)$.

The WBC count increased in all patients in both groups reaching a maximum count of $14.29 \times 10^{3} / \mathrm{CC}$ in group (A) (range $10-22 \times 10^{3} / \mathrm{CC}$ ) peaking at the $6^{\text {th }} \mathrm{PO}$ day and $12.65 \times 10^{3} / \mathrm{CC}$ in group (B) (range $11-15 \times 10^{3} / \mathrm{CC}$ ) peaking at the $3^{\text {rd }}$ PO day. The difference between the 2 groups was statistically significant $(\mathrm{P}<0.0012) \quad$ The platelet count increased in all patients reaching its peak at the $6^{\text {th }} \mathrm{PO}$ day; in group (A), the maximum platelet count was $620 \times 10^{3} / \mathrm{cc}$ (range $120-900 \times 10^{3} / \mathrm{CC}$ ) while in group (B), it was $690 \times 10^{3} / \mathrm{CC}$ (range $120-760 \times 10^{3} / \mathrm{CC}$ ). The difference between the 2 groups was statistically insignificant $(\mathrm{P}<0.5000)$

All patients with PS-PVT had complete resolution of the thrombus with recanalization of the PV and SMV on anticoagulation therapy within 3-6 months PO. None of these patients suffered upper GI bleeding, intestinal ischaemia, refractory ascites, liver cell failure or haemorrhagic complications secondary to anticoagulants. There was no mortality.

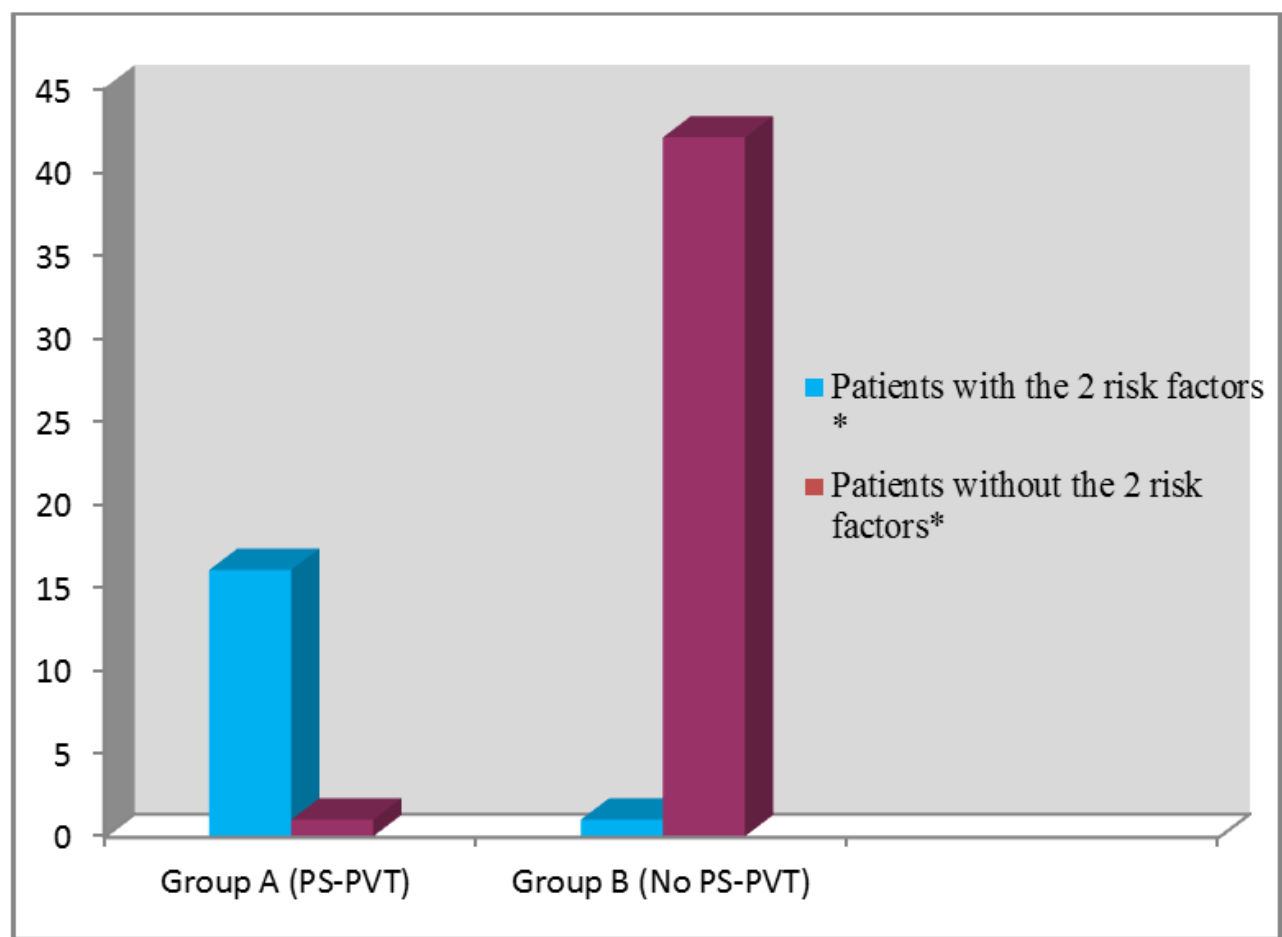

* Low preoperative white blood cell count and wide splenic vein diameter. PS-PVT: post-splenectomy portal venous thrombosis

Figure 1. Incidence of PS-PVT in Patients with and Without Low Preoperative WBC Count and Wide SVD.

\section{Discussion}

Portal vein thrombosis (PVT) is a frequent complication of splenectomy with a reported incidence of up to $50 \%$ in cirrhotic patients $[14,21]$. Several risk factors have been suggested in different studies [10-14, 21].

In this study, we observed a cohort of cirrhotic patients submitted to open splenectomy, both clinically and by Duplex US, looking for the development of this complication. We relied on Duplex Doppler US for the diagnosis of PS-PVT. Color Duplex US imaging has a specificity of $99 \%$ and a sensitivity of $93 \%$, while contrastenhanced computed tomography (CT) is also highly specific (99\%) but slightly less sensitive (90\%) [22]. Advantages of CT over US include the possibility of detecting bowel ischemia and septic foci and higher sensitivity in the detection of partial venous thrombosis and thrombosis in the $\mathrm{SV}$ and SMV. The drawbacks of CT include exposure to ionizing radiation, the risk of allergic reactions and nephrotoxicity [23].

The results of our study show that low WBC count $\left(<4 \times 10^{3} / \mathrm{CC}\right)$ and dilated splenic vein (diameter $\left.>13 \mathrm{~mm}\right)$ are independent risk factors for the development of PS-PVT in cirrhotic patients and patients who had these 2 variables showed a very high incidence of PS-PVT (94\%) in comparison to a very low incidence of $2.3 \%$ in those patients without these 2 variables. Our results agree with those of some studies $[12,14,20,21]$ while other studies did not identify these 2 variables as risk factors for PS-PVT [10, 13].

After splenectomy of large spleen, PVT has been suggested to be due to the DSV acting as a low flow cul-desac in which stagnation of blood flow leads to thrombosis which then spreads into the PV [24]. Some authors recommend ligation of the splenic vein as close as possible to its junction with the inferior mesenteric vein (IMV) so that the stagnant segment of the SV is removed while blood keeps flowing from the IMV into the remaining segment of the SV [25].

The WBC count in our study increased in all patients PO 
and the increase was significantly higher in group (A); a difference that represents a result rather than a cause of PVT. Although platelets are famous for their role in thrombus formation, there is significant evidence supporting an integral role for WBC in thrombosis and implying that leucocytosis may have a prothrombotic effect. Neutrophil-derived enzymes inhibit anticoagulants, such as tissue factor inhibitor, antithrombin III and Protein C [26-28] while activating factor XII and increasing expression of tissue factor $[26,27]$. Oxidants released by neutrophils can inhibit degradation of von Willebrand factor producing a prothrombotic state [29]. Impaired degradation of neutrophil extracellular traps has been associated with acute thrombotic microangiopathies [30]. It was also found that neutrophils and monocytes are major components of thrombi formed at sites of flow restriction and that the arrival of leucocytes, in large quantities, preceded the formation of a thrombus, suggesting their early role in thrombosis. When leucocytes were depleted in mouse models, thrombosis either did not occur or the thrombi were significantly smaller [31].

About $70 \%$ of patients who developed PS-PVT in our study had symptoms including anorexia, abdominal pain and distension. Because many patients remain asymptomatic, the true incidence of PS-PVT may be underestimated [32] and suspicion of PVT should be entertained in any patient who develops vague and generalized abdominal pain, fever or leukocytosis within the first 30 days after splenectomy; manifestations that are quite nonspecific, vague and insidious in onset and therefore unhelpful in establishing the diagnosis [33].

Although the median interval between splenectomy and PVT in the current study was 4.5 days, an important observation is that PVT was diagnosed $<24$ hours after splenectomy in 1 patient $(1 / 17 ; 5.9 \%)$. This observation underlines the importance of early screening in high risk patients. The median time from splenectomy to symptomatic PVT was $6,10.7,11.6$ and 12 days PO in other study [19, 32, 34].

All patient with PS-PVT in our study received anticoagulation therapy immediately after the diagnosis was established and all of them had complete resolution of the thrombus in PV and SMV. It was reported that recanalization may occur in $>90 \%$ of patients with acute PVT who are anticoagulated [35]. Rhee et al [36] demonstrated higher survival rates in patients with PVT who were anticoagulated compared with patients who were observed. In the van't Riet et al [19] series, all patients treated within 10 days of splenectomy had resolution of the clot versus no patient in whom treatment was initiated more than 10 days.

In our study, the preoperative platelet count was higher in group (A) than in group (B) patients and the difference between the 2 groups reached statistical significance on univariate analysis. On multivariate analysis, however, the difference failed to reach a statistical significance (OR: 0.958, 95\% CI: 0.125-1.652, $\mathrm{P}<0.095)$. Platelet count increased in all patients $\mathrm{PO}$, but, the difference between the 2 groups did not reach a statistical significance on univariate analysis. The association between post-splenectomy thrombocytosis and PS-PVT is unclear. Not all patients with post-splenectomy thrombocytosis develop PVT, and this complication has been described in patients with normal or even low platelet counts [37]. Therefore, postoperative thrombocytosis, even if markedly elevated, cannot be considered the only cause of PVT [38].

The small sample size is one of the limitations of this study. Also, some variables which were identified as risk factors for PS-PVT as splenic weight and anti-thrombin III were not assessed in our study.

\section{Conclusion}

PS-PVT is a common complication in cirrhotic patients occurring in $28.3 \%$ of patients in the current study. Low preoperative WBC count and wide SVD were identified as independent risk factors of PS-PVT. Close observation, both clinically and by Duplex US, starting from the $1^{\text {st }} \mathrm{PO}$ day and immediate anticoagulation after diagnosis yield excellent outcomes.

\section{Conflict of Interest Statement}

All the authors do not have any possible conflicts of interest.

\section{References}

[1] Shepard CW, Finelli L, Alter MJ (2005) Global epidemiology of hepatitis C virus infection. Lancet Infect Dis 5:558-67.

[2] Sievert W, Altraif I, Razavi HA, et al (2011) A systematic review of hepatitis $\mathrm{C}$ virus epidemiology in Asia, Australia and Egypt. Liver Int 31:61-80.

[3] El-Zanaty and Associates (2015) Egypt health issues survey 2015. Cairo, Egypt and Rockville, Maryland, USA: Ministry of Health and Population and ICF International 25-49.

[4] Struthers A (2007) From schistosomiasis to hepatitis C: the spread of HCV in Egypt. Med J Ther Africa 1(3):213-21.

[5] Darwish MA, Faris R, Darwish N, et al (2001) Hepatitis C and cirrhotic liver disease in the Nile delta of Egypt: A community-based study Am J Trop. Med Hyg 64:147-53.

[6] Kercher KW, Carbonell AM, Heniford BT, Matthews BD, Cunningham DM, Reindollar RW (2004) Laparoscopic splenectomy reverses thrombocytopenia in patients with hepatitis C cirrhosis and portal hypertension. $J$ Gastrointest Surg 8:120-6.

[7] Hirooka M, Ishida K, Kisaka Y, et al (2008) Efficacy of splenectomy for hypersplenic patients with advanced hepatocellular carcinoma. Hepatol Res 38:1172-7.

[8] Morihara D, Kobayashi M, Ikeda K (2009) Effectiveness of combination therapy of splenectomy and long-term interferon in patients with hepatitis C virus-related cirrhosis and thrombocytopenia. Hepatol Res 39:439-47. 
[9] Hassab MA, Younis MT, El-Kilany MS (1986) Gastroesophageal decongestion and splenectomy in the treatment of esophageal varices secondary to bilharzial cirrhosis: anatomical and experimental studies. Surg 63:73137.

[10] Li MX, Zhang XF, Liu ZW, Lv Y (2013) Risk factors and clinical characteristics of portal vein thrombosis after splenectomy in patients with liver cirrhosis. Hepatobiliary Pancreat Dis Int 12:512-519.

[11] Yoshida M, Watanabe Y, Horiuchi A, Yamamoto Y, Sugishita H, Kawachi K (2009) Portal and splenic venous thrombosis after splenectomy in patients with hypersplenism. Hepatogastroenterol 56(90):538-41.

[12] Kawanaka H, Akahoshi T, Tomikawa M et al (2010) Risk factors for portal venous thrombosis after splenectomy in patients with cirrhosis and portal hypertension. British J Surg 97:910-916.

[13] Jiang G, Bai D, Chen P, Qian J, Jin S, Wang X (2016) Risk Factors for portal vein system thrombosis after laparoscopic splenectomy in cirrhotic patients with hypersplenism. $J$ laparoendosc \& adv surg techn 26(6):419-423.

[14] Wu S, Wu Z, Zhang X, Wang R, Bai J (2015) The incidence and risk factors of portal vein system thrombosis after splenectomy and pericardial devascularization. Turk $J$ Gastroenterol 26:423-428.

[15] Stamou KM, Toutouzas KG, Kekis PB (2006) Prospective study of the incidence and risk factors of postsplenectomy thrombosis of the portal, mesenteric, and splenic veins. Arch Surg 141:663-669.

[16] Pietrabissa A, Moretto C, Antonelli G, Morelli L, Marciano E, Mosc F (2004) Thrombosis in the portal venous system after elective laparoscopic splenectomy. Surg Endosc 18:11401143.

[17] Winslow ER, Klingensmith ME, Brunt LM (2005) Problem of portal venous thrombosis after splenectomy. Ann Surg 242:745.

[18] Miniati DN, Padidar AM, Kee ST, Krummel TM (2005) Mallory B. Portal vein thrombosis after laparoscopic splenectomy: an ongoing clinical challenge. JSLS 9:335-8.

[19] Van't Riet M, Burger JWA, van Muiswinkel JM, Kazemier G, Schipperus MR, Bonjer HJ (2000) Diagnosis and treatment of portal vein thrombosis following splenectomy. $\mathrm{Br} J$ Surg 87:1229-33

[20] Kawanaka H, Akahoshi T, Itoh S, et al (2014) Decreased antithrombin III (ATIII) activity and large splenic vein diameter (SVD) Optimizing Risk Stratification in Portal Vein Thrombosis after Splenectomy and its Primary Prophylaxis with Antithrombin III Concentrates and Danaparoid Sodium in Liver Cirrhosis with Portal Hypertension. J Am Coll Surg 219:865-74.

[21] Danno K, Ikeda M, Sekimoto M, et al (2009) Diameter of splenic vein is a risk factor for portal or splenic vein thrombosis after laparoscopic splenectomy. Surgery 145:45764.

[22] Bach AM, Hann LE, Brown KT, et al (1996) Portal vein evaluation with US: comparison to angiography combined with CT arterial portography. Radiology 201:149-54.

[23] Margini C, Berzigotti A (2017) Portal vein thrombosis: The role of imaging in the clinical setting. Digestive and Liver Disease 49:113-20.

[24] Eguchi A, Hashizume M, Kitano S, Tanoue K, Wada H, Sugimachi K (1991) High rate of portal vein thrombosis after splenectomy in patients with esophageal varices and idiopathic portal hypertension. Arch Surg 126:752-55.

[25] Boissel P, Bresler L, Trackeon G, Poussot D, Gosdidier (1991) Mesenteric and portal vein thrombosis following splenectomy in hematologic diseases. A report of 3 cases. Chirurgie 117:591-95.

[26] Borissoff JI, ten Cate H (2011) From neutrophil extracellular traps release to thrombosis: an overshooting host-defense mechanism? J Thromb Haemost 9:1791-94.

[27] Massberg S, Grahl L, von Bruehl ML, et al (2010) Reciprocal coupling of coagulation and innate immunity via neutrophil serine proteases. Nat Med 16:887-96.

[28] Levi M, van der Poll T, Buller HR (2004) Bidirectional relation between inflammation and coagulation. Circulation 109:2698-704.

[29] Ruggeri ZM (2007) The role of von Willebrand factor in thrombus formation. Thromb Res 120 (Suppl 1):S5-S9.

[30] Jimenez-Alcazar M, Napirei M, Panda R, et al (2015) Impaired DNase1-mediated degradation of neutrophil extracellular traps is associated with acute thrombotic microangiopathies. J Thromb Haemost 732-42.

[31] von Bruhl ML, Stark K, Steinhart A, et al (2012) Monocytes, neutrophils, and platelets cooperate to initiate and propagate venous thrombosis in mice in vivo. J Exp Med 209:819-35.

[32] Romano F, Caprotti R, Conti M, et al (2006) Thrombosis of the splenoportal axis after splenectomy. Langenbecks Arch Surg 391:483-88.

[33] Benoist S (2000) Les complications a moyen et long term de la splenectomie. Ann Chir 125:317-24.

[34] Ikeda M, Sekimoto M, Takiguchi S, et al (2007) Total splenic vein thrombosis after laparoscopic splenectomy: a possible candidate for treatment. Am J Surg 193:21-5.

[35] Condat B, Pessione F, Denninger MH, et al (2000) Recent portal or portal vein thrombosis: increased recognition and frequent recanalization on anticoagulant therapy. Hepatology 32:466-70.

[36] Rhee RY, Gloviczki P, Mendonca CT, et al (1994) Portal vein thrombosis: still a lethal disease in the 1990s. J Vasc Surg 20:688-97.

[37] Winslow ER, Brunt LM, Drebin JA, Soper NJ, Klingensmith ME (2002) Portal vein thrombosis after splenectomy. Am J Surg 184:631-6.

[38] Rossi P, Passariello R, Simonetti. G (1976) Portal Thrombosis: High Incidence Following Splenectomy for Portal Hypertension. Gastrointest Radiol 1:225-7. 